\title{
Positive and negative patterning in human classical skin conductance response conditioning
}

\author{
HARALD LACHNIT \\ University of Marburg, Marburg, Germany \\ and \\ HERBERT D. KIMMEL \\ El Paso, Texas
}

\begin{abstract}
Three experiments on classical differential conditioning of the human skin conductance response to elemental and compound stimuli are reported. Subjects in Experiment 1 received both positive and negative patterning training, followed by either positive or negative patterning transfer tests on new stimuli. In positive patterning, a compound of two stimuli is reinforced and its elements are nonreinforced. In negative patterning, the elements are reinforced and the compound is nonreinforced. Subjects in Experiments 2 and 3 received either positive or negative patterning during training, followed by transfer tests on new stimuli. In Experiment 2, the transfer series began with new elements, after which their compound was presented; in Experiment 3, the new compound was presented first in the transfer series, and then the separate elements were administered. All three experiments provided evidence of the acquisition of positive patterning, while negative patterning was found only in Experiments 2 and 3. Positive patterning transferred to new stimuli, indicating that it was not attributable solely to summation of subthreshold excitation conditioned to the elements on reinforced compound trials. This finding, coupled with the negative patterning found in Experiments 2 and 3, provided support for the unique cue hypothesis. It was concluded that the assumed unique cue constituted a learned "rule," and that the actual elemental stimuli were neither perceptually nor otherwise modified during the conditioning process.
\end{abstract}

This report describes three studies of positive and negative patterning in human classical skin conductance response (SCR) conditioning. These studies attempted to determine whether the reinforcement rules involved in the acquisition of positive and negative patterning would transfer to new stimuli. Positive patterning involves a differentiation between a reinforced compound stimulus and its separately nonreinforced elements, while negative patterning involves a differentiation between a nonreinforced compound and its separately reinforced elements. The results of the present research were also expected to have relevance for current theoretical analyses of Pavlovian conditioning with elemental and compound stimuli.

Theories of Pavlovian conditioning, with infrequent exceptions (e.g., Pearce, 1987; Pearce \& Wilson, 1990), have treated compound stimuli in an atomistic fashion. This atomistic approach assumes that responding to a compound reflects summation of the separate associative strengths of the compound's constituent elements (Mack-

Our thanks are extended to Helmut Kleinschmitt, Regina Rettenbach, and Jörg Wolter for assistance in conducting the experiment. We also thank V. M. LoLordo and two anonymous reviewers for valuable commentary and criticism. Address correspondence concerning this article to Harald Lachnit, Department of Psychology, University of Marburg, Gutenbergstr. 18, D-35032 Marburg, Germany. intosh, 1975; Pearce \& Hall, 1980; Rescorla \& Wagner, 1972). Empirical outcomes in accord with summation of elements have frequently been obtained in studies of compound conditioning and stimulus compounding (Kehoe \& Gormezano, 1980). However, there is also abundant evidence that conflicts with an atomistic interpretation of responding to compound stimuli. The atomistic approach cannot account straightforwardly either for Saavedra's (1975) demonstration of biconditional discrimination or for the phenomena of positive and negative patterning. Saavedra presented four elemental stimuli, A, B, C, and $D$, equally often in reinforced and nonreinforced compounds. Compounds $\mathrm{AC}$ and $\mathrm{BD}$ were reinforced while $A B$ and $C D$ were not reinforced. Under these conditions, an observed difference in responding to the reinforced and nonreinforced compounds cannot be attributed to summation of excitation and/or inhibition associated with their constituent elements. Nevertheless, Saavedra's subjects learned to differentiate reinforced from nonreinforced compounds. This outcome obviously cannot be explained solely on the basis of excitation and inhibition conditioned to the separate elements. As noted above, positive-patterning subjects learn to differentiate nonreinforced elemental stimuli from their reinforced compound, whereas negative-patterning subjects learn the reverse differentiation. Although an atomistic approach can be made to accommodate positivepatterning results by assuming that subthreshold excitation 
conditioned to the elements on reinforced compound trials summates to elevate responding to the compound in comparison with either element separately, negative-patterning outcomes cannot readily be dealt with atomistically, since the positive associative strengths of the separate elements cannot combine additively to yield nonresponding to the compound.

A variety of theoretical proposals have been made in an effort to deal with these problems. Most of these approaches retain the atomistic summation principle but, in addition, assume that the compound contains an additional (unobserved) stimulus that is also sensitive to the reinforcement (or nonreinforcement) operations. The best known version of this type of "unique-cue" hypothesis was originally proposed by Rescorla $(1972,1973)$ and by Whitlow and Wagner (1972). It assumes that a compound, say, AB, consists of its elements, A and B, plus an additional hypothetical stimulus, $\mathrm{X}$, that is present when $\mathrm{A}$ and $B$ are administered simultaneously but not when either A or B is administered alone. This hypothetical stimulus can itself acquire the excitatory or inhibitory associative properties necessary to account for positive and negative patterning or biconditional discriminations. The RescorlaWagner (1972) model of Pavlovian conditioning, supplemented by the assumed unique-cue hypothesis, correctly predicts that reinforcement of $\mathrm{ABX}$ and nonreinforcement of A and B when either is presented alone will result in positive patterning (since $X$ is always associated with reinforcement, whereas $A$ and $B$ occur in conjunction with both reinforcement and nonreinforcement). The model, likewise, predicts negative patterning, since nonreinforcement of $A B X$ is here pitted against reinforcement of $\mathrm{A}$ and $\mathrm{B}$ ( $\mathrm{X}$ is always associated with nonreinforcement, whereas $A$ and $B$ are paired with both reinforcement and nonreinforcement).

A study by Rescorla, Grau, and Durlach (1985) attempted to determine whether the hypothetical unique cue is better thought of as resulting from a primitive perceptual interaction between the elemental stimuli, that is, as a Gestalt or configural stimulus, or as "a rule in which the joint activation of representations of A and B generates a stimulus"' (Rescorla et al., 1985, p. 357). Using an autoshaping procedure in pigeons, they administered negativepatterning training involving $\mathrm{A}+, \mathrm{B}+$, and $\mathrm{AB}-$, and then used second-order conditioning to pair two new stimuli, $X$ and $Y$, with $A$ and $B$, respectively. The pigeons then correctly differentiated $X$ and $Y$ from their compound, XY. Rescorla et al. concluded from these results that the unique cue assumed to be present along with the $\mathrm{AB}$ compound during the original negative-patterning training could not have depended upon the sensory-perceptual properties of $A$ and $B$, since it would then not have been activated when $\mathrm{X}$ and $\mathrm{Y}$ were presented subsequently as a compound. The physical presence of the stimuli involved in the originally trained differentiation was not necessary for the subjects to demonstrate that they had learned negative patterning. Thus, the pigeons behaved as if they had learned a "rule," namely, that compounds consisting of reinforced elements are not reinforced (or that elemental stimuli are positive, whereas compound stimuli are negative). The second-order conditioning procedure mediated the transfer of this rule from the originally trained $A$ and $\mathrm{B}$ to $\mathrm{X}$ and $\mathrm{Y}$, respectively.

In the present research, a somewhat different postacquisition "transfer" strategy was used to evaluate the unique-cue concept in both positive and negative patterning. Like Rescorla et al. (1985), we examined transfer of patterning to stimuli different from those used in the original differential conditioning. But, instead of using second-order conditioning to establish an association between the originally trained stimuli and the to-be-tested transfer stimuli, we followed the initial patterning training with two training trials on two new elemental stimuli, and then tested the compound containing these new stimuli (Experiments 1 and 2), or we followed the initial training with two presentations of a compound containing two new stimuli, and then presented the elements alone (Experiment 3). Like Rescorla et al., we assumed that, during the initial patterning series, the subject might learn a "rule" which could function as a unique cue. The transfer tests were intended to provide evidence of whether the subjects could apply the "rule" assumed to have been learned during the original compound-element differentiation to the new stimuli. Evidence of transfer of a compound-element differentiation to new stimuli would provide support for the assumption of the unique-cue hypothesis.

To examine transfer of positive patterning, the new elemental stimuli were presented twice without reinforcement before the compound containing these elements was tested (Experiments 1 and 2), or a compound containing two new stimuli was presented twice with reinforcement before its nonreinforced elements were tested (Experiment 3). This permitted us to separate a simple summation explanation of positive patterning from one involving a unique cue (a rule). If the subjects acquired the positive patterning differentiation and transferred it to the new stimuli (by responding more to the new compound than to its elements), this could not be due to summation of excitation conditioned to the new elements, since these elements were never reinforced. Furthermore, transfer of positive patterning would also mean that the unique cue assumed to be present during the acquisition of the original compound-element differentation was more like a rule than a perceptual Gestalt, since the transfer test involved totally different stimuli from those used in the initial differentiation. Experiments 2 and 3 differed only in the order in which the compound and elements occurred during the transfer series, so that the role of the orienting reflex elicited by the novel transfer stimuli could also be assessed.

The transfer procedure following negative-patterning training was administered in the same way as for positive patterning, except that the elements were reinforced and the compound was not. If the behavior of our subjects was governed by the assumed "rule," responding should have been less to the new compound on the transfer test 
than to its elements. Summation of excitation from the reinforced new elements to their compound, on the other hand, would yield the opposite result. Transfer of negative patterning would also support the assumption that the unique cue involved during the acquisition of negative patterning was more like a rule than a Gestalt configural stimulus. Again, Experiments 2 and 3 differed only with respect to the order of compound and element stimuli in the transfer series.

\section{EXPERIMENT 1}

In Experiment 1, 40 human subjects received an SCR conditioning procedure involving both positive and negative patterning, each of these being associated with a different pair of stimuli. Thirty of these subjects then received a negative-patterning transfer test on new stimuli; 10 subjects received a positive-patterning transfer test on new stimuli. The decision to employ more subjects for the negative-patterning transfer test was based upon previous findings (Bellingham, Gillette-Bellingham, \& Kehoe, 1985 ) suggesting that negative patterning is more difficult to learn than positive patterning and that the acquisition of negative patterning is even more difficult when subjects have previously experienced positive patterning. To "compensate" for the expected weaker negative-patterning effect, we administered the negative-patterning transfer test to the larger number of subjects (thus, providing greater sensitivity for this test).

On the basis of pilot study data indicating that human subjects can acquire the present positive and negative rules at a verbal level, it was hypothesized that SCR magnitudes would reflect the acquisition of both the positive- and negative-patterning rules; that is, that the compound would elicit larger SCRs than its constituent elements for stimuli given positive-patterning training and smaller SCRs than its constituent elements for stimuli given negativepatterning training. It was further expected that the transfer tests would reflect the application of these rules to new stimuli. As noted above, the positive-patterning rule was that a compound containing elements that are not followed by electric shock is itself followed by shock; the negativepatterning rule was that a compound consisting of elements that are followed by shock is itself not followed by shock. Since the subject received training relevant to both of these rules during the same training session, it was also possible that a supraordinate rule could be acquired, combining the two lower order rules. That is, it was possible for the subject to learn the rule that compounds and their elements always have opposite valence; if the elements are shocked, the compound is not, and if the compound is shocked, the elements are not. Also, the administration of both positive and negative patterning in the same session meant that the subject could not rely on a "two positive-one negative" or "two negative-one positive" rule. The subject had to employ either different (opposite) rules for positive and negative patterning or the supraordinate rule described above.

\section{Method}

Subjects and Instructions. Forty University of Giessen students (17 males and 23 females, mean age $=23.7$ years) volunteered to serve as subjects (some to meet course requirements). They were assigned randomly to three groups ( $n=10, n=10$, and $n=20$ ). Data from 5 additional subjects were discarded due to equipment errors or failure to follow instructions (excessive movement and/or heavy breathing). The subjects were informed that the purpose of the experiment was to measure their physiological responses to various stimuli, including letters projected on a screen and occasional electric shocks, and that they were to relax and avoid unnecessary movement and heavy breathing. They were also told to pay close attention to the stimuli, since they would later have to complete a questionnaire regarding what had occurred.

Apparatus. Six letters were employed as conditioned stimuli (CSs), M, J, C, N, L, and V. They were presented by a slide projector on a screen $1.50 \mathrm{~m}$ in front of the subject's face. The letters were $15 \mathrm{~cm}$ high and had a maximum width of $10 \mathrm{~cm}$. The letters had a duration of $8 \mathrm{sec}$. A direct current electric shock served as unconditioned stimulus (US). The shock was delivered via silversilver chloride electrodes to the volar surface of the subject's left arm from an isolated transformer-condensor shock generator (Kimmel, King, Hudy, \& Gardner, 1980). The subjects adjusted the intensity of the shock themselves so that it would be "definitely unpleasant but not really painful." Intensities ranging from 1.0 to $4.0 \mathrm{~mA}$ resulted. Shock duration was $10 \mathrm{msec}$. On paired conditioning trials, the shock was delivered simultaneously with CS offset (i.e., a delayed conditioning paradigm with a CS-US interval of $8 \mathrm{sec}$ was employed). The intertrial interval during training and transfer testing was $33 \mathrm{sec} \pm 3 \mathrm{sec}$ (CS onset to CS onset). The administration and timing of the stimuli were computer controlled. Palmar skin conductance was picked up from the thenar and hypothenar eminences of the subject's right hand by $0.8-\mathrm{cm}$-diam silversilver chloride electrodes filled with an electrolytic medium (Unibase, with $.05 \mathrm{M} \mathrm{NaCl}$ ). The skin was cleaned with alcohol prior to electrode attachment. Skin conductance signal conditioning was accomplished by means of a constant-voltage bridge described by Lykken and Venables (1971) and sampled by the computer at $20 \mathrm{~Hz}$.

Procedure. Data were collected in a sound-attenuated, electrically shielded room, illuminated by flourescent lighting. The subject sat in a cushioned chair facing the projector screen. When the subject arrived at the laboratory, he/she was seated in the experimental chamber and the electrodes were attached. The subject then read the written instructions and was asked if they were understood. If the subject did not understand the instructions, they were presented again. The initial conditioning procedure consisted of 30 trials that contained five presentations each of the letters $M, J, C$, and $N$, and five presentations each of the compounds $\mathrm{MJ}$ and $\mathrm{CN}$. On compound trials, the elements were presented simultaneously. The stimuli were administered in blocks of six trials, such that each of the elements and compounds occurred once within each block. The training sequence was designed to facilitate acquisition of the positiveand negative-patterning rules (e.g., $\mathrm{M}+, \mathrm{J}+, \mathrm{MJ}-, \mathrm{C}-, \mathrm{N}-$, $\mathrm{CN}+, \mathrm{N}-, \mathrm{CN}+, \mathrm{C}-, \mathrm{M}+, \mathrm{MJ}-, \mathrm{J}+$, etc.), except that no more than three successive reinforced or nonreinforced stimuli were administered. For one group of 10 subjects, $M$ and $J$ were reinforced, MJ was nonreinforced, $\mathrm{C}$ and $\mathrm{N}$ were nonreinforced, and $\mathrm{CN}$ was reinforced. Thus, for these 10 subjects, $\mathbf{M}, \mathbf{J}$, and $\mathrm{MJ}$ were trained according to a negative-patterning arrangement, while $\mathrm{C}, \mathrm{N}$, and $\mathrm{CN}$ were trained according to a positive-patterning arrangement. Following the initial training sequence, this group received two rein- 
forced presentations each of two new stimuli, L and V, followed by a nonreinforced test trial on LV. Thus, this group received a negative-patterning transfer test on LV. A second group of 10 subjects received the same positive- and negative-patterning training as did the first group, except that their training sequence began with the nonreinforced $\mathrm{C}$ and $\mathrm{N}$. The second group then received two nonreinforced presentations of $\mathrm{L}$ and $\mathrm{V}$, followed by a nonreinforced positive-patterning transfer test on $\mathrm{LV}$. The remaining 20 subjects received a conditioning sequence that was identical to that given to the second group, and then, following initial training, L and $V$ with reinforcement and a nonreinforced negative-patterning transfer test on LV. Thus, 30 of the $\mathbf{4 0}$ subjects received a negativepatterning transfer test, while 10 had a positive-patterning transfer test. At the conclusion of the conditioning procedure, the subjects completed a questionnaire and were thanked and dismissed.

Dependent variables. The SCR was defined as the maximum conductance change occurring during the interval 1-4 sec after CS onset. These conductance changes were converted to logarithmic values (after adding 1), as recommended by Venables and Christie (1980), and multiplied by 1,000 .

\section{Results}

The .05 significance level was employed in all statistical tests, and stated probability levels are based on the Greenhouse-Geisser (1959) adjustment of degrees of freedom where appropriate.

Initial acquisition. Mean SCR magnitudes for the separate elements and their compounds during the initial 30 conditioning trials are presented in Figure 1 (left panel, positive patterning; right panel, negative patterning). In both panels, responses on trials involving elements are averaged in two-trial blocks, while responses on the corresponding compound trials are presented singly. Since

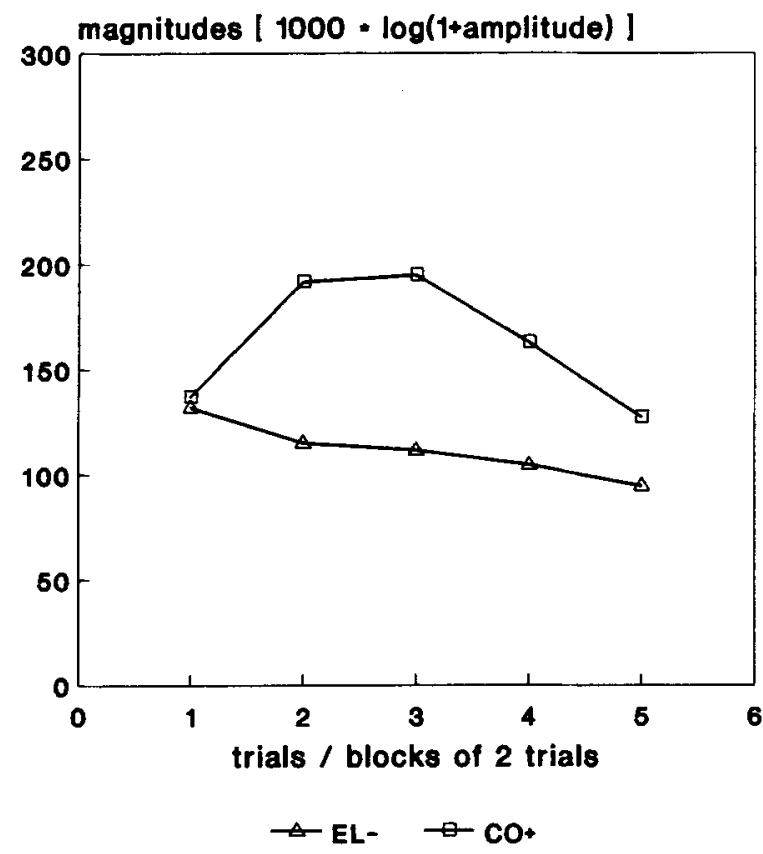

all 40 subjects received a combination of positive- and negative-patterning conditioning procedures (differing only in order of stimuli), their acquisition data are combined in both panels. Positive patterning, as shown in the left panel of Figure 1, developed relatively rapidly, with responses to the compound clearly being larger than those to the elements at the second data point. Analysis of variance (ANOVA) of the positive patterning data indicated that the mean difference in SCR magnitudes in response to the compound and elements was significant $[F(1,39)=$ $29.43, p<.001]$ and that the interaction of the compoundelement difference and trial blocks was also significant $[F(4,156)=3.37, p<.01]$.

The negative-patterning data are shown in the right panel of Figure 1. As is clear in the figure, negative patterning did not develop. Indeed, there did not appear to be an increase in magnitude of responding to either the elements or the compound. An ANOVA confirmed that there was no negative-patterning effect $[F(1,39)=.02]$ and that the interaction with trial blocks was also insignificant $[F(4,156)=1.12]$. Thus, when subjects were given a combination of both positive- and negative-patterning training, clear positive patterning was found in the SCR but negative patterning did not occur.

Transfer test. Following only two conditioning trials on each of two new elements, their compound was tested without reinforcement. For the positive-patterning transfer test, the new stimuli were presented twice each without reinforcement, followed by a nonreinforced presentation of their compound. An atomistic theory implies either that responding to these elements and their compound should

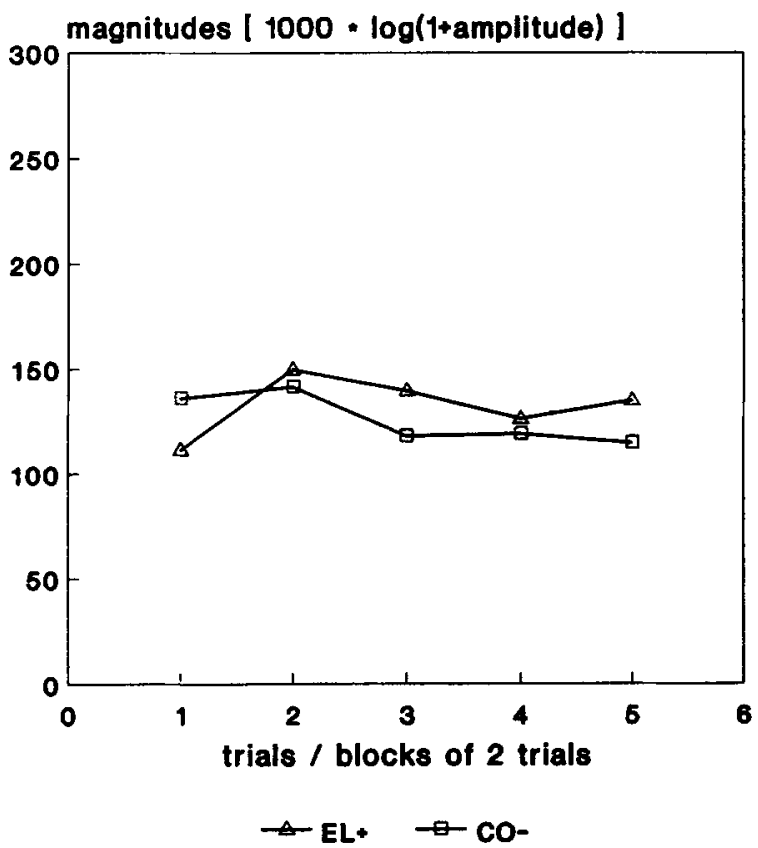

Figure 1. Mean magnitudes of skin conductance responses during initial acquisition of Experiment 1. In positive patterning (left panel), elements were nonreinforced ( $\mathrm{EL}-$ ) and compounds were reinforced $(\mathrm{CO}+)$. In negative patterning (right panel), elements were reinforced $(\mathrm{EL}+$ ) while components were nonreinforced $(\mathrm{CO}-)$. Responses to elements are in two-trial blocks and to compounds in single trials. 
not differ (since the elements acquired no excitation) or that responding to the compound should be slightly smaller (due to summation of inhibition from the nonreinforced elements). For the negative-patterning transfer test, the new stimuli were presented twice each with shock followed by a nonreinforced presentation of their compound. Here an atomistic interpretation implies that responding to the compound should exceed that to the elements, due to the summation of excitation. Application of the uniquecue "rule" to the new stimuli in each of these cases should result in element-compound differences opposite to those predicted by an atomistic theory; that is, in the positivepatterning transfer test, responding to the compound should exceed that to the elements, whereas in the negativepatterning transfer test, responding to the compound should be smaller than that to the elements.

The empirical data relevant to these predictions are shown in Figure 2, with the positive-patterning transfer data in the left panel and the negative-patterning transfer data in the right panel. In Figure 2, the responses to the second presentation of each of the new elements were averaged for comparison with the response to the compound, because the subject had experienced both positive and negative patterning prior to the initial presentation of the transfer stimuli and, for this reason, had no basis for anticipating which of the two rules would apply to them. After the first presentation of each of the new stimuli, however, transfer of the rule exemplified by whether or not they were paired with shock could be examined

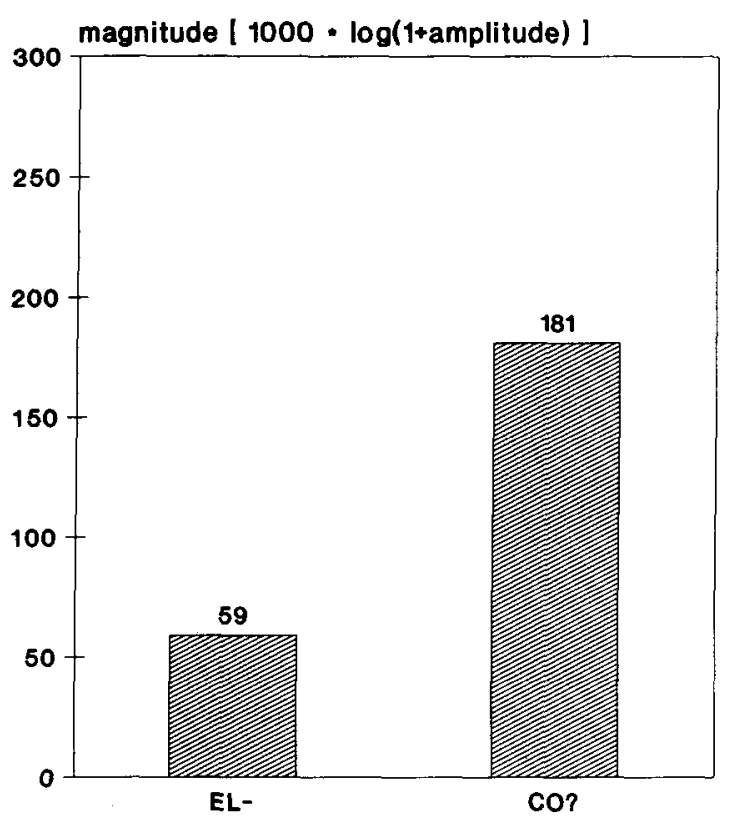

by comparing responses to their second presentations with the response to their compound.

The data shown in the left panel of Figure 2 suggest that the positive patterning observed during initial training transferred to the new stimuli. The transfer test trial responses to the compound were larger than those to the elements. The negative-patterning transfer data in the right panel of Figure 2, on the other hand, indicate that there was almost no difference in SCR magnitudes elicited by the elements and the compound. An ANOVA of the combined positive- and negative-patterning transfer data showed that the overall positive-negative-patterning difference was not significant $[F(1,38)=0.19]$, while the overall compound-element difference was significant $[F(1,38)=$ $6.66, p<.02]$. More importantly, the interaction between these two factors was significant $[F(1,38)=5.47, p<$ $.03]$. Separate evaluation of the positive- and negativepatterning transfer differences, however, showed a significant effect for neither positive patterning $[t(9)=1.94$, $p<.10]$ nor negative patterning $(t<1)$.

\section{Discussion}

The results of the initial acquisition phase of Experiment 1 provided only weak support for the unique-cue hypothesis. Although significant positive patterning was observed during initial acquisition, the negative-patterning differentiation was not significant. In addition, although the transfer tests revealed a significant interaction between the element-compound differences and positive versus

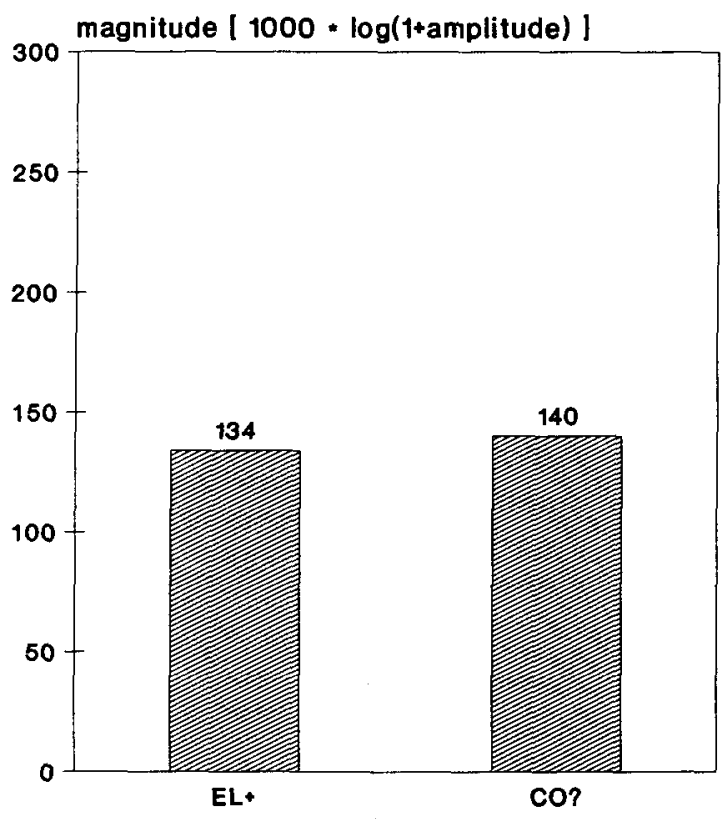

Figure 2. Mean magnitudes of skin conductance responses in transfer test trials of Experiment 1 for positive patterning (left panel) and negative patterning (right panel). In positive patterning, transfer test elements were nonreinforced (EL-) and the compound was tested (CO?). In negative patterning, transfer test elements were reinforced $(E L+)$ and the compound was tested (CO?). 
negative patterning in the direction predicted by the unique-cue hypothesis, separate tests of transfer of positive and negative patterning failed to attain significance.

The significant positive-patterning differentiation observed during initial acquisition cannot be taken as definitive evidence in support of the unique-cue hypothesis, since it is possible that larger responses to the reinforced compound in comparison with the elements may have been due to summation of excitation conditioned to the stimuli during the reinforced compound trials. Furthermore, the results of the transfer tests were also only weakly supportive of the unique-cue hypothesis. The combined analyses of transfer of positive and negative patterning, indicating a significant interaction between the compound-element and positive-negative-patterning factors, favored the unique-cue hypothesis over the atomistic approach. If responding during the transfer test trials depended solely upon the subject's experience on those trials (i.e., did not reflect transfer of what was learned during initial acquisition), the significant observed interaction between positivenegative patterning and compound-element stimuli would either have been absent or would have been in the reverse direction. Although it is true that an asymmetry existed in the way in which the transfer tests were conductedthat is, no shocks at all in the positive-patterning transfer series, while the elements were shocked in the negative patterning transfer series-an atomistic approach would suggest that responding to the compound in the positivepatterning transfer group would either not differ from responding to the elements or be slightly smaller, while responding to the compound in the negative-patterning transfer group should have been larger than that to the elements, based on summation of the excitation conditioned to them. The observed interaction in the transfer data cannot be made to fit this kind of atomistic approach but does agree with the assumption that a rule acquired in initial training was applied to the new stimuli.

It is also remotely possible that the observed transfer effect was methodologically biased because responses to the second presentation of the new elements were compared with responses to the first presentation of the new compound. To the extent that the orienting response to the novel transfer stimuli might have habituated on their initial presentations, they might have elicited smaller responses the second time they occurred. Then, if the compound elicited a renewed orienting response because of its novelty, the observed element- compound difference might not really have been due to the transfer of a rule learned during initial acquisition. This issue was considered further in Experiments 2 and 3.

Bellingham et al. (1985) have shown that negative patterning is more difficult to learn when it is preceded by positive patterning than when it is not. It therefore may be that the failure to obtain significant transfer of either positive or negative patterning separately may have been due to the simultaneous presence of both patterning rules during initial acquisition, that is, making them harder to learn. Stronger acquisition of the positive-patterning rule (and acquisition of the negative-patterning rule as well) might provide a better basis for the significant transfer of these rules to new stimuli. This question was examined empirically in Experiment 2.

\section{EXPERIMENT 2}

It is possible that both negative and positive patterning could be separately acquired and could transfer to new stimuli, if each of them was administered to separate groups of subjects. Experiment 2 focused on this question. One group of subjects received only positive-patterning training, and the other received only negative-patterning training. As in Experiment 1, two training trials were then administered on each of two new elements, followed by a transfer test on their compound. In the positive-patterning group, the new elements were presented without reinforcement, so that their transfer test evaluated transfer of positive patterning. In the negative-patterning group, the new elements were presented with reinforcement, so that their transfer test evaluated transfer of negative patterning. Because the subjects in Experiment 2 experienced only a single rule during initial acquisition (either positive or negative patterning), even the very first presentation of the transfer stimuli could be employed to assess the possibility of transfer (unlike Experiment 1, where there was no basis for predicting which rule applied to the new stimuli until after each of them had been presented once with or once without reinforcement). Thus, responses to all four administrations of the elemental transfer stimuli (two trials per stimulus) could be used to evaluate possible transfer effects. Also, because responses to the very first presentations of the novel transfer stimuli were employed in the transfer test, confounding of compoundelement orienting-response differences with the transfer difference were also less likely.

Since the initial training involved only positive or negative patterning, it was possible to employ a larger number of conditioning trials ( 8 on each element and 16 on their compound). The compound was administered twice as many times as either element in order to equate the two groups in terms of relative number of reinforced and nonreinforced trials (i.e., if the number of compound presentations had been the same as the number of presentations of either element, as in Experiment 1, the negativepatterning group would have had twice as many reinforced trials as did the positive-patterning group). This change in procedure increased the relative numbers of presentations of compounds versus elements (as compared with Experiment 1) and offered the possibility of enhanced acquisition of both positive and negative patterning.

\section{Method}

Subjects. Twenty University of Giessen students (11 males and 9 females, mean age $=23.8$ years) volunteered to serve as subjects. They were assigned randomly to two groups of 10 subjects
} 
each. Data from 4 additional subjects were discarded due to equipment errors or failure to follow instructions (excessive movement and/or heavy breathing). The instructions were identical to those of Experiment 1.

Apparatus and Stimuli. The apparatus, stimuli, and timing of stimuli and trials were the same as in Experiment 1 . Half of the subjects in each group received initial patterning training with the letters $\mathrm{M}$ and $\mathrm{J}$; the other half received $\mathrm{C}$ and $\mathrm{N}$. Following initial training, $\mathrm{L}$ and $\mathrm{V}$ were employed as new stimuli for transfer training and testing.

Procedure. Data were collected in the same chamber as in Experiment 1 . After arriving at the laboratory, the subject was seated in a cushioned chair facing the projector screen, the electrodes were attached, and the instructions were presented. The initial conditioning procedure consisted of 32 trials, containing 8 presentations each of the letters $\mathrm{M}$ and $\mathrm{J}$ (or $\mathrm{C}$ and $\mathrm{N}$ ) as elements, and 16 presentations of $\mathrm{MJ}$ (or $\mathrm{CN}$ ) as compound. The stimuli were administered quasi-randomly, with no more than three consecutive reinforced or nonreinforced stimuli (e.g., $\mathrm{J}-, \mathrm{MJ}+, \mathbf{M}-, \mathbf{M}-, \mathrm{J}-, \mathrm{MJ}+$, $M-, J-, M J+, M J+$, etc., in the positive-patterning group, and $\mathbf{J}+, \mathbf{M J}-, \mathbf{M}+, \mathbf{M}+, \mathbf{J}+, \mathbf{M J}-, \mathbf{M}+, \mathbf{J}+, \mathbf{M J}-, \mathbf{M J}-$, etc., in the negative-patterning group). Following the initial training sequence, each group received two presentations each of two new stimuli, $\mathrm{L}$ and $\mathrm{V}$, followed by a test trial on the $\mathrm{LV}$ compound. The elements were nonreinforced in the positive-patterning group but were reinforced in the negative-patterning group. All of the subjects completed the same questionnaire as in Experiment 1 and then were thanked and dismissed.

\section{Results}

The SCR was defined and transformed as in Experiment 1 . The .05 level was again used for significance tests, and degrees of freedom were Greenhouse-Geisser (1959) adjusted where appropriate.

\section{magnitudes $[1000 \cdot \log (t+a m p l i t u d e)]$}

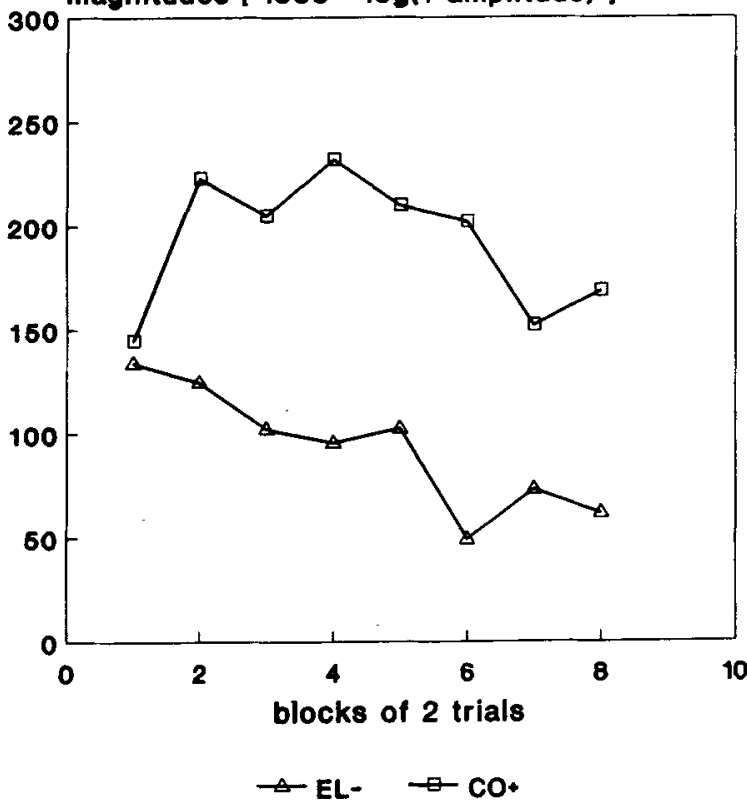

Initial acquisition. Mean SCR magnitudes for responses to the elements and compound during the initial 32 conditioning trials are presented in Figure 3 (left panel, positive patteming; right panel, negative patterning), averaged in two-trial blocks for the elements and compounds. Positive patterning developed very rapidly, as is shown in the left panel of Figure 3. Responses to the reinforced compound were substantially larger than those to the nonreinforced elements, beginning with the second trial block. An ANOVA of the positive-patterning data indicated that the mean difference in SCR magnitude in response to the compound and elements was significant $[F(1,9)=24.48$, $p<.001]$. The interaction between the compound-element difference and trial blocks was not significant $[F(7,63)=$ 1.73]. The negative-patterning data are shown in the right panel of Figure 3. As can be seen in this figure, negative patterning was again (as in Experiment 1) not readily acquired, even when its acquisition was not complicated by the simultaneous presence of positive-patterning training. After some 4-5 blocks of training trials, however, a difference in mean SCR to the reinforced elements and nonreinforced compound appeared, and persisted for the remainder of the session. An ANOVA of the data shown in the right panel of Figure 3 indicated that the compoundelement difference was statistically significant $[F(1,9)=$ $13.23, p<.01]$. The apparent interaction between the compound-element difference and trial blocks, however, was not significant $(F<1)$.

Transfer test. Following two conditioning trials on each of two new elements, their compound was tested. In the case of positive patterning, the two new letters were

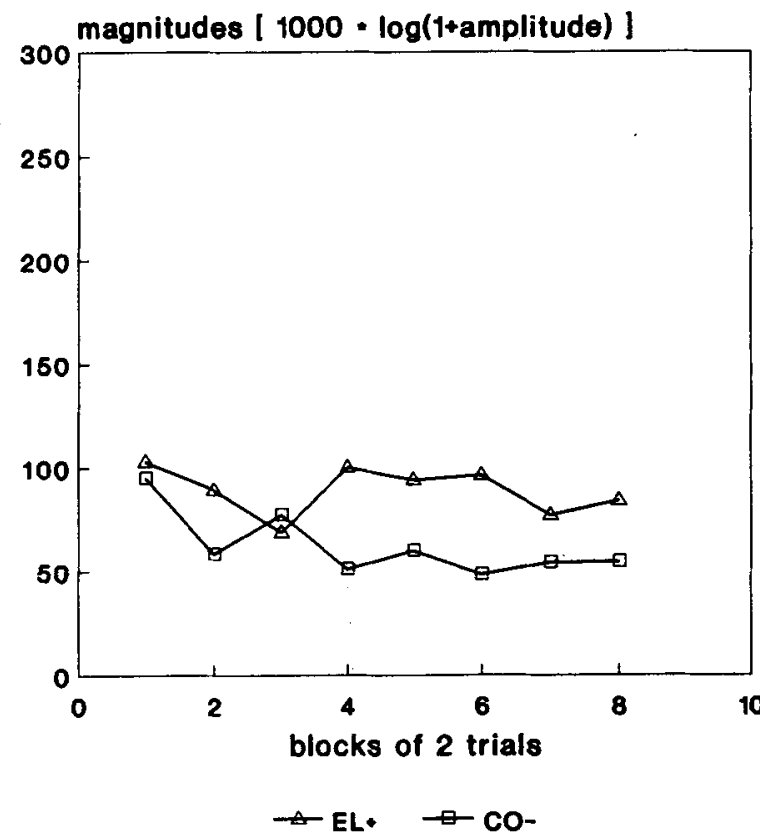

Figure 3. Mean magnitudes of skin conductance responses during initial acquisition of Experiment 2. In positive patterning (left panel), elements were nonreinforced $(\mathrm{EL}-)$ and compounds were reinforced $(\mathrm{CO}+)$. In negative patterning (right panel), elements were reinforced $(\mathrm{EL}+)$ while components were nonreinforced (CO-). Responses to elements and compounds are in two-trial blocks. 
presented without shock, followed by the compound. In the case of negative patterning, the new elements were presented with shock, followed by the compound. If the initial positive- and/or negative-patterning differentiations involved the acquisition of a rule that functioned as a unique cue, the acquired rule should have transferred to the new stimuli in the transfer test. Figure 4 presents the transfer test data, with the positive-patterning group in the left panel and the negative-patterning group in the right panel. In each panel of Figure 4, the average of the responses to the initial presentations of the two elemental transfer stimuli is shown on the left, the average of the responses to the second presentations of the elemental stimuli is in the middle, and the response to the compound is on the right. As can be seen in the left panel of Figure 4, responding to the transfer compound in the positivepatterning group was greater than the average response to the elements on both their first and second presentations. An ANOVA of the positive-patterning transfer data shown in the left panel of Figure 4 showed that the overall differences among the three means were significant $[F(2,18)=6.15, p<.02]$. Dunnett's test showed that the mean SCRs to the first and second presentations of the transfer elements both differed significantly from the response to the compound (.05 level critical difference $=94.1$ ). The right panel of Figure 4 shows that responding to the compound in the negative-patterning transfer test was smaller than that to either the first or second presentations of the elemental stimuli. An ANOVA of the

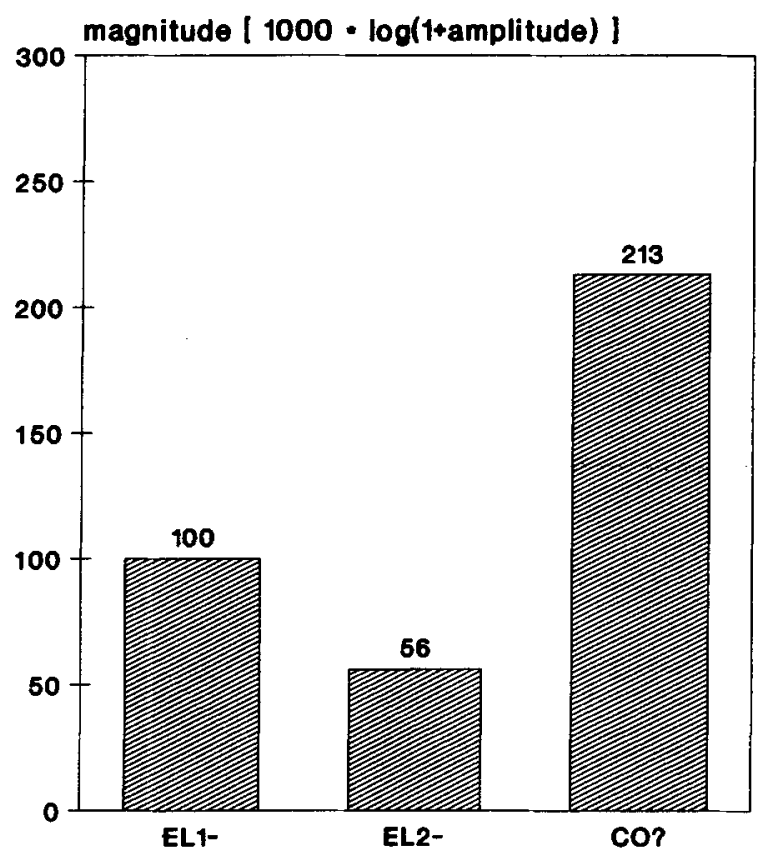

negative-patterning transfer data showed that the differences among the three means did not attain significance $[F(2,18)=2.86, p<.09]$. Dunnett's test showed that the difference between the mean SCR to the first presentation of the transfer elements and the compound was significant, but that the response to the second element did not differ significantly from that to the compound (.05 level critical difference $=52.3$ ) .

\section{Discussion}

The results of Experiment 2 provided evidence of the acquisition of both positive and negative patterning in the human SCR preparation. Significant differentiations between a reinforced compound and its nonreinforced elements (positive patterning) and between a nonreinforced compound and its reinforced elements (negative patterning) both occurred. In addition, significant transfer of positive patterning was observed when responses to either the first or second administrations of the transfer elements were compared with responses to their compound. When compared with the transfer results in Experiment 1, where only the second presentations of the transfer elements could be used in the transfer test, these findings suggest that orienting-response differences were not responsible for the observed positive-patterning transfer effects. The response to the transfer compound was significantly greater than that to the first presentation of the new transfer elements, even though it may be assumed that the new elements were at least as surprising to the subject as the

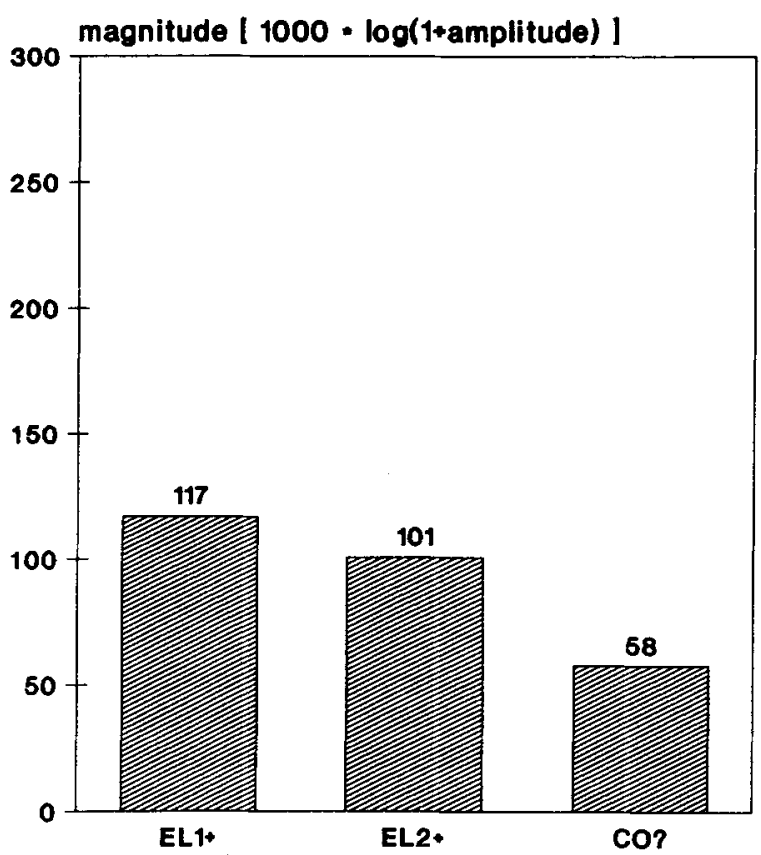

Figure 4. Mean magnitudes of skin conductance responses in transfer test trials of Experiment 2 for positive patterning (left panel) and negative patterning (right panel). In positive patterning, transfer test elements were nonreinforced (EL1 -, first presentation of two elements; ELL - , second presentation of two elements) and the compound was tested (CO?). In negative patterning, transfer test elements were reinforced (EL1 +, first presentation of two elements; EL2 +, second presentation of two elements) and the compound was tested (CO?). 
new compound. Significant transfer of negative patterning was also found in Experiment 2, but only when responses to the initial presentations of the transfer elements were compared with responses to their compound. Even though these initial presentations of the negative-patterning transfer stimuli were reinforced with shock, responses on the second administrations of the transfer stimuli were not also significantly larger than responses to their compound, as might have been expected. This complication was analyzed further in Experiment 3, where the order of the administration of elemental and compound stimuli was reversed in the transfer series.

Although significant acquisition of negative patterning was obtained in Experiment 2, this difference did not develop until relatively late in conditioning. Thus, the fact that the subjects in the negative-patterning group experienced only negative patterning (unlike Experiment 1, where both rules were present) may not have been the only factor contributing to this outcome. The additional training trials administered in Experiment 2 and/or the increased number of compound trials relative to element trials as compared with Experiment 1, may also have facilitated the acquisition of the negative-patterning differentiation (as compared with Experiment 1). When responses to only the first 10 elements and five compounds are examined (there were only 10 elements and five compounds in the negative patterning comparison in Experiment 1), the negative-patterning differentiation in Experiment 2 was virtually nonexistent.

In discussing the results of Experiment 1, we pointed out that the observed significant interaction between the compound-element and positive-negative-patterning factors in the overall ANOVA of the transfer data did not, in itself, constitute persuasive evidence in support of the unique-cue hypothesis, because the positive- and negativepatterning transfer tests differed somewhat in procedure and because neither effect transferred significantly when tested separately. The finding of significant transfer of positive patterning when either the initial or second trial elements were compared with the compound in Experiment 2, however, provides strong support for the conclusion that the positive-patterning differentiation that developed during initial training was due to the acquisition of a rule. The new elements were presented twice without reinforcement, and then their compound was presented. Excitation from the new elements could hardly have summated to elevate responding to the transfer compound, since the new elements were never reinforced. Transfer of the rule acquired during initial acquisition to the new stimuli, on the other hand, would have led to the observed differences.

As noted previously, the very acquisition of negative patterning, in itself, can be viewed as evidence in favor of the unique-cue hypothesis, whether or not transfer of negative patterning to new stimuli occurs. In Experiment 2 , there was some evidence of the transfer of negative patterning to new stimuli, but only when responses to the initial presentations of these stimuli were compared with responses to their compound. The fact that the second-trial responses were not significantly larger than the responses to the compound in the negative-patterning transfer test indicated that further examination of the possibility of confounding of order effects with the transfer effects was necessary. In Experiment 3, the order of compound and elemental stimuli in the transfer series was reversed from that used in Experiments 1 and 2.

\section{EXPERIMENT 3}

The purpose of Experiment 3 was to determine whether the order of presentation of elemental and compound stimuli in the transfer series might have contributed to the observed transfer effects. In addition, Experiment 3 constituted an exact replication of the positive- and negativepatterning acquisition procedures of Experiment 2. Since the positive- and negative-patterning differentiations were both statistically significant in Experiment 2, when different groups of subjects received training in one but not both of these differential conditioning procedures, it was of interest to determine whether both differentiations would again be observed in an independent sample of subjects.

\section{Method}

Subjects. Twenty University of Giessen students (11 males and 9 females, mean age $=24.7$ years) volunteered to serve as subjects. They were assigned randomly to the positive- and negativepatterning groups and received the same instructions as in Experiment 2 .

Apparatus and Stimuli. The apparatus, stimuli, and timing of stimuli and trials were the same as in Experiment 2. Half of the subjects in each group received initial patterning training with the letters $\mathrm{M}$ and $\mathrm{J}$; the other half received $\mathrm{C}$ and $\mathrm{N}$. Following initial training, $L$ and $V$ were employed as new stimuli for transfer training and testing.

Procedure. Data were collected in the same chamber as in Experiments 1 and 2 . After arriving at the laboratory, the subject was seated in a cushioned chair facing the projector screen, the electrodes were attached, and the instructions were presented. The initial conditioning procedure consisted of 32 trials, containing 8 presentations each of the letters $M$ and $J$ (or $C$ and $N$ ) as elements, and 16 presentations of $\mathrm{MJ}$ (or $\mathrm{CN}$ ) as compound. The stimuli were administered quasi-randomly, with no more than three consecutive reinforced or nonreinforced stimuli (e.g., $\mathbf{J}-, \mathbf{M J}+, \mathbf{M}-, \mathbf{M}-$, $\mathrm{J}-, \mathrm{MJ}+, \mathbf{M}-, \mathrm{J}-, \mathrm{MJ}+, \mathbf{M J}+$, etc., in the positive-patterning group, and $\mathbf{J}+, \mathbf{M J}-, \mathbf{M}+, \mathbf{M}+, \mathbf{J}+, \mathbf{M J}-, \mathbf{M}+, \mathbf{J}+, \mathbf{M J}-$, $\mathrm{MJ}-$, etc., in the negative-patterning group). Following the initial training series on either positive or negative patterning, the transfer series began with two presentations of a compound containing two new stimuli, $L$ and $V$. In the positive-patterning group, the compound was paired with shock, while in the negative-patterning group, the compound was presented without shock. Then each of the new elemental stimuli was presented once alone, paired with shock in the negative-patterning group and presented without shock in the positive-patterning group. Two presentations of the compound were employed instead of only one as in Experiment 2, in order to achieve balance with the Experiment 2 transfer series, which had begun with two presentations of the new elements. At the conclusion of the transfer series, the subject completed the same questionnaire, as in Experiments 1 and 2 and then was thanked and dismissed. 

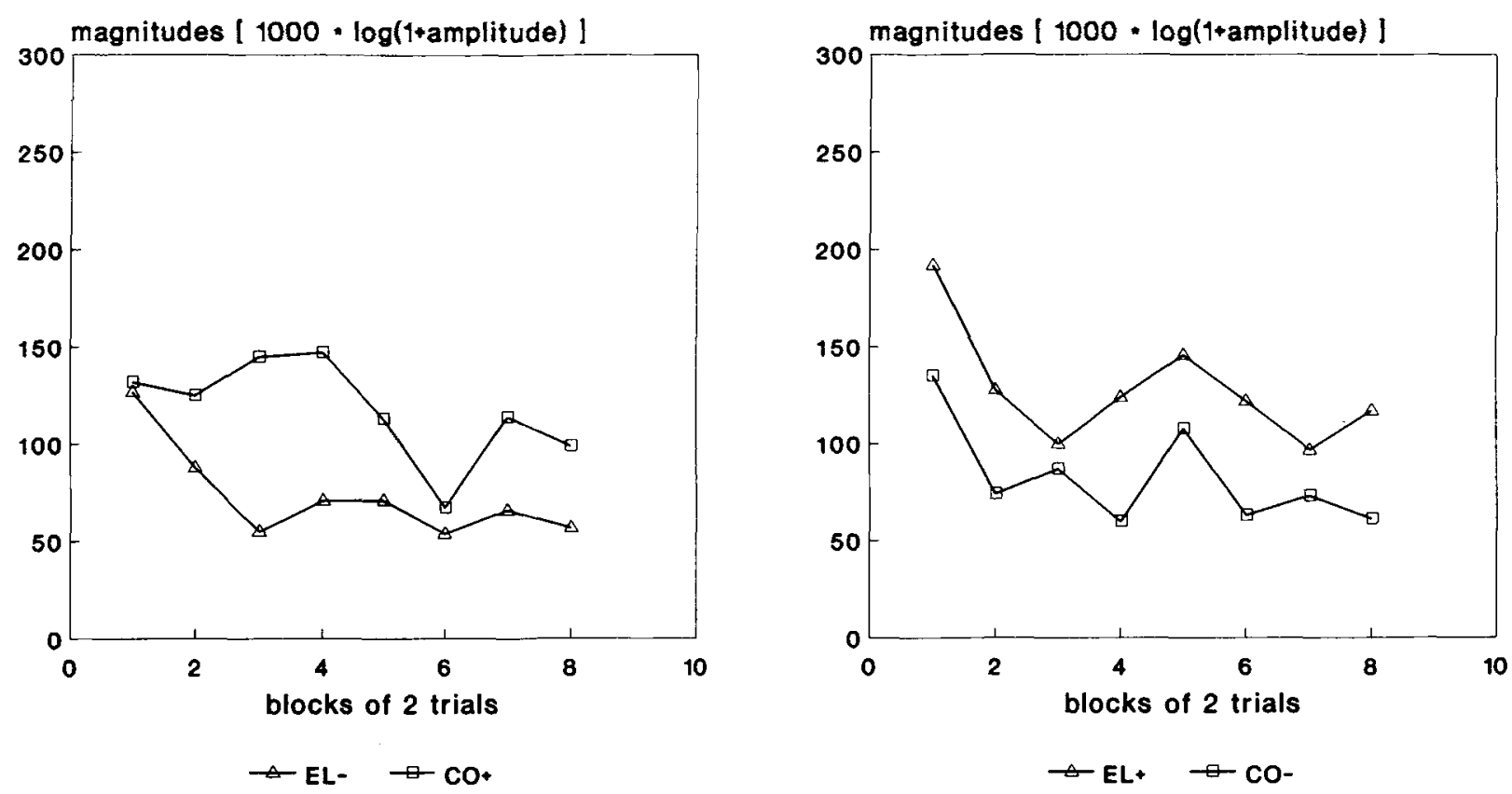

Figure 5. Mean magnitudes of skin conductance responses during initial acquisition of Experiment 3. In positive patterning (left panel), elements were nonreinforced $(\mathrm{EL}-)$ and compounds were reinforced $(\mathrm{CO}+)$. In negative patterning (right panel), elements were reinforced $(\mathrm{EL}+)$ and components were nonreinforced $(\mathrm{CO}-)$. Responses to elements and compounds are in two-trial blocks.

\section{Results}

The SCR was defined and transformed as in Experiments 1 and 2 . The .05 level was again used for significance tests, and degrees of freedom were GreenhouseGeisser (1959) adjusted where appropriate.

Initial acquisition. Mean SCR magnitudes for responses to the elements and compound during the initial 32 conditioning trials are presented in Figure 5 (left panel, positive patterning; right panel, negative patterning), averaged in two-trial blocks for the elements and compounds. As in Experiment 2, positive patterning again developed very rapidly, as shown in the left panel of Figure 5. Responses to the reinforced compound were substantially larger than those to the nonreinforced elements, beginning with the second trial block. An ANOVA of the positive-patterning data indicated that the mean difference in SCR magnitude in response to the compound and elements was significant $[F(1,9)=11.98, p<.008]$. The interaction between the compound-element difference and trial blocks was not significant $[F(7,63)=1.51]$.

The negative-patterning data are shown in the right panel of Figure 5. As can be seen in this figure, negative patterning was quite readily acquired (unlike in Experiment 2). The mean SCR was larger to the reinforced elements than to the nonreinforced compound from the very first block of training trials. An ANOVA of the data shown in the right panel of Figure 5 indicated that the compound-element difference was statistically significant $[F(1,9)=10.90, p<.01]$. The interaction between the compound-element difference and trial blocks, however, was not significant $(F<1)$.
Transfer test. The transfer series consisted of two presentations of a compound containing new stimuli, followed by one presentation of each of the elements. In the positivepatterning group, the new compound stimulus was paired with shock and the elements were not. In the negativepatterning group, the compound was presented without shock and the elements were paired with shock. If the initial positive- and/or negative-patterning differentiations involved the acquisition of a rule that functioned as a unique cue, the acquired rule should have transferred to the new stimuli in the transfer test.

Figure 6 presents the transfer test data, with the positivepatterning group in the left panel and the negative-patterning group in the right panel. In each panel of Figure 6, the average of the responses to the initial presentations of the new compound is shown on the left, the average of the responses to the second presentations of the compound is in the middle, and the average of the responses to the two elements is on the right. As can be seen in the left panel of Figure 6, responding of the positive-patterning group was greater to both the first and second presentations of the transfer compound than was their average response to the elements. An ANOVA of the positive-patterning transfer data shown in the left panel of Figure 6 showed that the overall differences among the three means were significant $[F(2,18)=5.54, p<.04]$. Dunnett's test showed that the mean SCRs to the first presentation of the compound differed significantly from the response to the elements (.05 level critical difference $=76.8)$, but the response to the second presentation of the compound was not significantly larger than the response to the ele- 

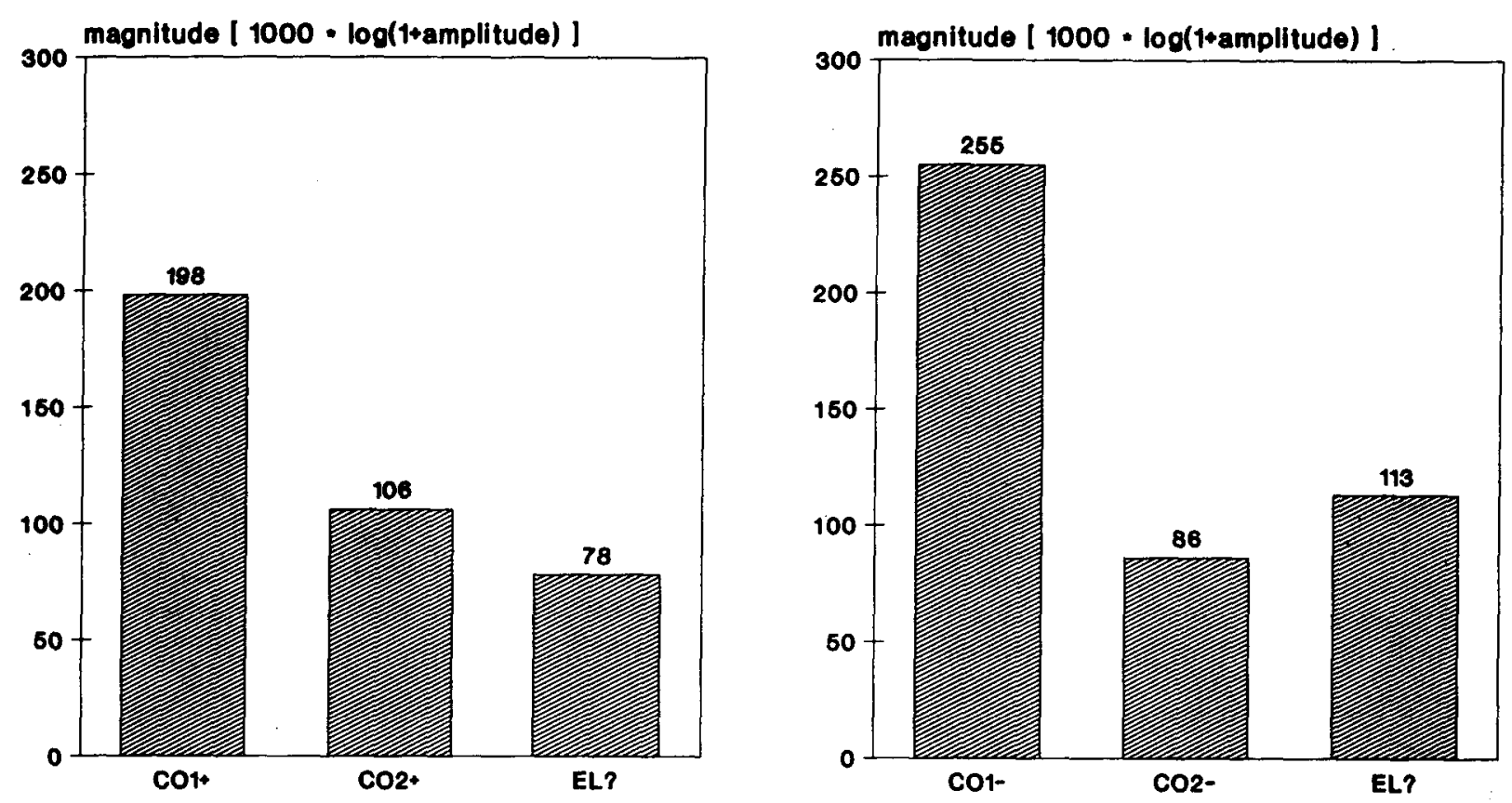

Figure 6. Mean magnitudes of skin conductance responses in transfer test trials of Experiment 3 for positive patterning (left panel) and negative patterning (right panel). In positive patterning, transfer test elements were nonreinforced (CO1 + , first presentation of the compound; $\mathrm{CO} 2+$, second presentation of the compound) and the element was tested (EL?). In negative patterning, transfer test compounds were reinforced ( $\mathrm{CO} 1-$, first presentation of two compounds; $\mathrm{CO}$-, second presentation of two compounds) and the element was tested (EL?).

ments. The right panel of Figure 6 shows that responding to the first presentation of the compound in the negativepatterning transfer test was larger than the response to the elements, while the response to the second presentation of the compound was smaller than the response to the elements. An ANOVA of the negative-patterning transfer data showed that the overall differences among the three means were significant $[F(2,18)=7.27, p<$ $.02]$. Dunnett's test showed that the reversed difference between the mean SCR to the first presentation of the compound and the mean SCR to the elements was significant ( .05 level critical difference $=97.0$ ) and the response to the second compound did not differ significantly from the response to the elements.

\section{Discussion}

The main purpose of Experiment 3 was to determine whether the transfer effects observed in Experiment 2 depended upon the order in which the new elemental stimuli and their compound were administered, that is, to examine the possibility that orienting responses to unexpected stimuli might have contributed to the transfer differences. The positive-patterning transfer data showed that responding was significantly greater to the first presentation of a compound containing new stimuli than it was to the subsequently presented separate elements. The shock that was paired with this compound CS did not occur until after the SCR to the CS was elicited. The corresponding dif- ference in the positive-patterning transfer test of Experiment 2 was also significant, even though the order of compound and elemental stimuli was reversed in the two experiments. Application of the positive-patterning rule acquired during initial conditioning to the new stimuli in the transfer series appears to be the most likely basis for the positive-patterning transfer effects in both Experiment 2 and Experiment 3, since the order of administration of elemental and compound stimuli did not affect the observed effect.

The negative-patterning transfer test, on the other hand, yielded a compound-element difference opposite to what would have been expected if the negative-patterning rule employed during initial training had been applied to the new stimuli. Indeed, the negative-patterning transfer test data of Experiments 2 and 3 appeared to reflect the influence of an orienting reaction to novelty, since the SCR on the first transfer trial was greater than that on subsequent trials, regardless of whether the first trial consisted of an elemental stimulus or a compound. Experiment 3 also was an exact replication of the positive- and negativepatterning initial training procedures of Experiment 2 with an independent sample of subjects. The observed positiveand negative-patterning acquisition differences were both again statistically significant. It is, thus, abundantly clear that human subjects can acquire compound-element Pavlovian differentiations on the basis of differential aversive reinforcement. 


\section{GENERAL DISCUSSION}

To the extent that the positive- and negative-patterning rules can be conceptualized as unique cues, in the sense that this concept has been employed by Rescorla et al. (1985) and Whitlow and Wagner (1972), the results of the present studies provide evidence of the usefulness of this extension of the Rescorla-Wagner (1972) theory for positive as well as negative patterning. Significant positive patterning was observed in Experiments 1, 2, and 3, and significant negative patterning was observed in Experiments 2 and 3. Furthermore, significant transfer of positive patterning was found in Experiments 2 and 3 independent of the order of presentation of the elemental and compound stimuli. There was evidence of transfer of negative patterning only when, in Experiment 2, the new elemental stimuli were presented first and their compound was administered subsequently. But, because this difference was reversed in Experiment 3, where the sequence of compound and elements was reversed, it is likely that the negative patterning transfer effect in Experiment 2 was due to the orienting response to the novel transfer elements.

The finding of significant positive patterning does not, in itself, require rejection of an atomistic explanation of responding to compound stimuli. But significant transfer of positive patterning to new stimuli clearly supports the assumption that a rule was acquired during the initial training and that this rule was then applied to the new stimuli in the transfer test. The fact that transfer of positive patterning was independent of the order of presentation of elemental and compound stimuli effectively rules out the possibility that it may have been due to differences between orienting reactions resulting from differences in stimulus novelty. As noted in the discussion of the results of Experiment 2, the elemental transfer stimuli were administered there without reinforcement prior to the presentation of the compound. Once an orienting-response interpretation of the positive-patterning transfer effect of Experiment 2 is ruled out by the finding that the same effect occurred in Experiment 3, where the order of elemental and compound stimuli was reversed, it is clear that the present positive-patterning differences reflect the acquisition of a rule rather than summation of excitation from the elements to the compound.

The significant acquisition of negative patterning in Experiments 2 and 3 obviously cannot be explained atomistically. Indeed, the phenomenon of negative patterning was one of the empirical problems confronting the RescorlaWagner theory that led to the formulation of the uniquecue hypothesis. The absence of unequivocal evidence of transfer of negative patterning to new stimuli does not seriously attenuate the negative impact of negative patterning on atomistic theories such as Rescorla-Wagner's. We assume that the present negative-patterning differentiation was a more difficult learning challenge to the sub- ject and, under the conditions of the present experiments, was not as readily acquired as was positive patterning. Thus, negative patterning did not transfer to new stimuli as readily either.

Comparison of the results of Experiment 1 with those of Experiments 2 and 3 suggests that both positive and negative patterning were acquired more readily when only one of them was administered to the subject. This conclusion was most clearly supported by the negativepatterning results, since there was no evidence at all for negative patterning in Experiment 1 but significant negative patterning was found in Experiments 2 and 3. The fact that there was significant evidence of transfer of positive patterning in Experiments 2 and 3 but not in Experiment 1 also is in accord with this conclusion. As noted above, however, Experiments 2 and 3 also contained a greater number of training trials than did Experiment 1, and twice as many trials on compounds as on either element. Thus, it is not altogether clear whether it was the fact that positive and negative patterning were both administered in Experiment 1 or that Experiment 1 had fewer training trials on both positive and negative patterning and fewer compound training trials per element training trial that was responsible for the weaker acquisition and lack of transfer in Experiment 1.

The design of the present series of studies was expressly influenced by Rescorla et al.'s (1985) demonstration that the stimuli in their original negative-patterning differentiation training did not need to be physically present for the acquired negative-patterning rule to be demonstrated. However, to the extent that the second-order CSs employed in their transfer tests can be assumed to have evoked some kind of representation of the original stimuli (due to their association with them via second-order conditioning), it might be argued that the unique cue was still indirectly tied to the originally trained stimuli. Thus, their results provided only limited support for the conclusion that a learned rule underlay the observed negative patterning. The present demonstration of the transfer of positive patterning to new stimuli that were neither reinforced nor associated with the originally trained stimuli is not susceptible to this criticism, however. In this respect, the present results agree with and go beyond those of Rescorla et al. in demonstrating the inadequacy of atomistic explanations of responding to compound stimuli and in providing support for the unique-cue hypothesis. Thus, our conclusion is that the subjects in the present series of experiments learned to respond differentially to the compound and elemental stimuli because these stimuli exemplified either positive- or negative-patterning rules. Significant transfer of the positive-patterning rule to new stimuli, then, indicated that the rule rather than the particular stimuli employed during its acquisition governed the observed differentiations. We assume that the negativepatterning rule, being more difficult, was not learned strongly enough to be applied to the transfer stimuli. 


\section{REFERENCES}

Bellingham, W. P., Gillette-Bellingham, K., \& Kehoe, E. J. (1985). Summation and configuration in patterning schedules with the rat and rabbit. Animal Learning \& Behavior, 13, 152-164.

Greenhouse, S. W., \& Geisser, S. (1959). On methods in the analysis of profile data. Psychometrika, 24, 95-112.

Kenoe, E. J., \& Gormezano, I. (1980). Configuration and combination laws in conditioning with compound stimuli. Psychological Bulletin, 87, 351-378.

Kimmel, H. D., King, J., Hudy, J. J., \& Gardner, K. A. (1980). A mutual inductance shocker. Behavior Research Methods \& Instrumentation, 12, 605-606.

LYKKEN, D. T., \& VENABLES, P. H. (1971). Direct measurement of skin conductance: A proposal for standardization. Psychophysiology, 8, 656-672.

Mackintosh, N. J. (1975). A theory of attention: Variation in the associability of stimuli with reinforcement. Psychological Review, 82, 276-298.

Pearce, J. M. (1987). A model of stimulus generalization for Pavlovian conditioning. Psychological Review, 94, 61-73.

Pearce, J. M. , Hale, G. (1980). A model for Pavlovian conditioning: Variations in the effectiveness of conditioned but not of unconditioned stimuli. Psychological Review, 7, 532-552.

PearCe, J. M., \& Wilson, P. N. (1990). Configural associations in discrimination learning. Journal of Experimental Psychology: Animal Behavior Processes, 16, 250-261.
Rescorla, R. A. (1972). “Configural" conditioning in discrete-trial bar-pressing. Journal of Comparative \& Physiological Psychology, 79, 307-317.

ResCORLA, R. A. (1973). Evidence for a unique-cue account of configural conditioning. Journal of Comparative \& Physiological Psychology, 85, 331-338.

Rescorla, R. A., Grau, J. W., \& Durlach, P. J. (1985). Analysis of the unique cue in configural discriminations. Joumal of Experimental Psychology: Animal Behavior Processes, 11, 356-366.

Rescorla, R. A., \& WAGNer, A. R. (1972). A theory of Pavlovian conditioning: Variations in the effectiveness of reinforcement and nonreinforcement. In A. Black \& W. F. Prokasy (Eds.), Classical conditioning II (pp. 64-99). New York: Appleton-Century-Crofts.

SaAvedra, M. A. (1975). Pavlovian compound conditioning in the rabbit. Learning \& Motivation, 6, 314-326.

Venables, P. H., \& CHristie, M. J. (1980). Electrodermal activity. In I. Martin \& P. H. Venables (Eds.), Techniques in psychophysiology (pp. 4-67). Chichester, U.K.: Wiley.

Whitlow, J. W., \& WAGNER, A. R. (1972). Negative patterning in classical conditioning: Summation of response tendencies to isolable and configural components. Psychonomic Science, 27, 299-301.

(Manuscript received November 2, 1991; revision accepted for publication March 20, 1993.) 